\title{
On the meta distribution in spatially correlated non-Poisson cellular networks
}

\author{
Shanshan Wang ${ }^{*}$ and Marco Di Renzo (i)
}

\begin{abstract}
In this paper, we consider a cellular network in which the locations of the base stations are spatially correlated. We introduce an analytical framework for computing the distribution of the conditional coverage probability given the point process, which is referred to as the meta distribution and provides one with fine-grained information on the performance of cellular networks beyond spatial averages. To this end, we approximate, from the typical user standpoint, the spatially correlated (non-Poisson) cellular network with an inhomogeneous Poisson point process. In addition, we employ a new and recently proposed definition of the coverage probability and introduce an efficient numerical method for computing the meta distribution. The accuracy of the proposed approach is validated with the aid of numerical simulations.
\end{abstract}

Keywords: Cellular networks, Stochastic geometry, Inhomogeneous Poisson point processes, Meta distribution

\section{Methods/experimental}

The methods used in the present paper are based on the mathematical tools of inhomogeneous Poisson point processes and stochastic geometry. A new analytical framework for performance analysis is introduced. The theoretical framework is validated against Monte Carlo simulations.

\section{Introduction}

Stochastic geometry and point processes are well known and widely used analytical tools for modeling, analyzing, and optimizing cellular networks [1]. The Poisson Point Process (PPP), in particular, is the most widely used spatial model to describe the locations of the base stations (BSs) in cellular networks [2-6]. This is due to its inherent analytical tractability. In practice, however, the locations of cellular BSs are distributed according to complex spatial patterns that are difficult to model analytically and, in general, differ from the PPP [7].

To overcome the analytical complexity of modeling and analyzing non-Poisson, i.e., spatially correlated, cellular networks, we have introduced an approximation based on inhomogeneous PPP, which is referred to as inhomogeneous double thinning (IDT) approximation [7]. The

${ }^{*}$ Correspondence: shanshan.wang@telecom-paristech.fr Laboratoire des Signaux et Systèmes, CNRS, CentraleSupelec, Univ Paris-Sud, Université Paris-Saclay, Plateau du Moulon, 91192 Gif-sur-Yvette, France
IDT approach allows one to model both spatially repulsive and spatially attractive (clustered) cellular network deployments in a mathematically tractable manner. In [7], in particular, the suitability of the IDT approach has been tested against several cellular network deployments obtained from publicly available datasets, and a good accuracy has been obtained.

In [7], however, the IDT approach is used to compute the spatially averaged coverage probability in cellular networks. More precisely, the coverage probability is first formulated by conditioning upon the point process that describes the locations of the cellular BSs, and then the expectation with respect to the point process is computed. As remarked in [8], the spatially averaged coverage probability is an important performance metric, but it does not completely characterize the variability of the coverage probability around its (spatial) mean value. To overcome this limitation, the author of [8] has introduced the concept of meta distribution, which provides one with finer-grained information on the network performance. Notably, the meta distribution allows one to characterize the performance of user percentiles. More precisely, the coverage probability is first formulated by conditioning upon the point process that describes the locations of the cellular BSs, and, then, its complementary cumulative distribution function with respect to the point process is computed. The spatially averaged coverage probability 
can be retrieved from the meta distribution via a simple integration.

In the present paper, motivated by these considerations and by [9], we generalize the IDT approach for computing the meta distribution in non-Poisson cellular networks. We consider a recent and improved definition of the coverage probability, which allows one to account for the signal quality during the cell association and data transmission phases [10], [11]. We show, notably, that the meta distribution cannot be approximated, in general, by using the beta distribution. As remarked in [12-14], however, the numerical computation of the meta distribution is usually not straightforward by employing the Gil-Pelaez inversion theorem [15]. We prove, on the other hand, that the meta distribution can be efficiently computed by employing the trapezoidal integration rule and the Euler sum method, for which a bound for the approximation error is known [16, 17]. With the proposed approach, the meta distribution in spatially correlated (non-Poisson) cellular networks can be obtained efficiently and accurately. The suitability of the proposed approach is substantiated with the aid of numerical simulations.

The reminder of this paper is organized as follows. In Section 3, the system model and the IDT approach are introduced. In Section 4, the meta distribution is formulated and an efficient method for its computation is given. In Section 5, analytical expressions for the moments of the coverage probability conditioned upon the point process that describes the locations of cellular BSs are derived. In Section 6, Poisson and non-Poisson cellular networks are compared against each other. In Section 7, numerical simulations to validate the suitability and accuracy of the proposed approach are illustrated. Finally, Section 8 concludes this paper.

\section{System model}

In this section, we describe the system model and summarize the essence of the IDT approach for modeling non-Poisson cellular networks. The adopted definition of coverage probability is introduced as well.

\subsection{Cellular network modeling}

The cellular network model is the same as in [7]. In particular, a downlink cellular network is considered. The BSs are modeled as points of a motion-invariant point process, denoted by $\Psi_{\mathrm{BS}}$, of density $\lambda_{\mathrm{BS}}$. The locations of the BSs are denoted by $x \in \Psi_{\mathrm{BS}} \subseteq \mathbb{R}^{2}$. The mobile terminals (MTs) are distributed independently of each other and uniformly at random in $\mathbb{R}^{2}$. The density of the MTs is denoted by $\lambda_{\mathrm{MT}}$. A fully loaded assumption is considered, i.e., $\lambda_{\mathrm{MT}} \gg \lambda_{\mathrm{BS}}$, which implies that all the BSs are active and have MTs to serve. The BSs and MTs are equipped with a single omnidirectional antenna. Each BS transmits with a constant power denoted by $\mathrm{P}_{\mathrm{tx}}$. Thanks to the assumption of motion-invariance, the point process of the BSs is stationary and isotropic. As a result, the analytical frameworks are developed for the typical MT, denoted by $\mathrm{MT}_{0}$, that is located at the origin. The $\mathrm{BS}$ serving $\mathrm{MT}_{0}$ is denoted by $\mathrm{BS}_{0}$. Its location is denoted by $x_{0} \in \Psi_{\mathrm{BS}}$. All available BSs transmit on the same physical channel as $\mathrm{BS}_{0}$. The point process of the interfering $\mathrm{BSs}$ is denoted by $\Psi_{\mathrm{BS}}^{(\mathrm{I})}$, and the generic interfering BS is denoted by $\mathrm{BS}_{i}$. Besides the inter-cell interference, the Gaussian noise with power $\sigma_{\mathrm{N}}^{2}$ is taken into account as well.

All BS-to- $\mathrm{MT}_{0}$ links are assumed to be mutually independent and identically distributed (i.i.d.). For each BS-to$\mathrm{MT}_{0}$ link, path-loss and fast-fading channel impairments are considered. The path-loss is defined as $l(x)=\kappa\|x\|^{\gamma}$, where $\kappa$ and $\gamma>2$ are the path-loss constant and the path-loss slope (exponent), respectively. The power gain due to the fast-fading is assumed to follow an exponential distribution with mean 1 and is denoted by $g_{x}$ for $x \in \Psi_{\mathrm{BS}}$.

A cell association criterion based on the highest average received power is considered. Let $x \in \Psi_{\mathrm{BS}}$ be the location of a generic BS. The location, $x_{0}$, of the serving $\mathrm{BS}, \mathrm{BS}_{0}$, is obtained as follows:

$$
x_{0}=\underset{x \in \Psi_{\mathrm{BS}}}{\arg \max }\{1 / l(x)\}=\underset{x \in \Psi_{\mathrm{BS}}}{\arg \max }\left\{1 / L_{x}\right\}
$$

where $L_{x}=l(x)$ is a shorthand notation. As far as the intended link is concerned, we have $L_{0}=l\left(x_{0}\right)=$ $\min _{x \in \Psi_{B S}}\left\{L_{x}\right\}$.

\subsection{Coverage probability}

The definition of (spatially averaged) coverage probability recently introduced in [10] and [11] is considered. Let $\gamma_{D}$ and $\gamma_{A}$ be the reliability thresholds for the successful decoding of information data and for the successful detection of the serving $\mathrm{BS}, \mathrm{BS}_{0}$, respectively. The coverage probability, $\mathrm{P}_{\mathrm{cov}}$, of the typical MT, $\mathrm{MT}_{0}$, is as follows:

$$
\mathrm{P}_{\mathrm{Cov}}\left(\gamma_{\mathrm{D}}, \gamma_{\mathrm{A}}\right)=\operatorname{Pr}\left\{\mathrm{SIR} \geq \gamma_{\mathrm{D}}, \overline{\mathrm{SNR}} \geq \gamma_{\mathrm{A}}\right\}
$$

where the signal-to-interference ratio (SIR) and the average signal-to-noise ratio $(\overline{\mathrm{SNR}})$ can be formulated, for the network model under analysis, as follows:

$$
\begin{aligned}
& \mathrm{SIR}=\frac{\mathrm{P}_{\mathrm{tx}} g_{0} / L_{0}}{\sum_{\mathrm{BS}_{i} \in \Psi_{\mathrm{BS}}^{(\mathrm{I})}} \mathrm{P}_{\mathrm{tx}} g_{i} / L_{i} \mathbb{1}\left(L_{i}>L_{0}\right)} \\
& \overline{\mathrm{SNR}}=\frac{\mathrm{P}_{\mathrm{tx}} / L_{0}}{\sigma_{\mathrm{N}}^{2}}
\end{aligned}
$$

where $\mathbb{1}(\cdot)$ denotes the indicator function and $\overline{\mathrm{SNR}}$ is averaged with respect to the fast-fading power gain $g_{0}$ of the intended link.

It is worth mentioning that the definition of $\mathrm{P}_{\text {cov }}$ in (3) reduces to the conventional definition of coverage probability [1] by setting $\gamma_{\mathrm{A}}=0$. This implies that the results 
obtained in the present paper apply unaltered even if the conventional definition of coverage probability based only on the SIR is employed.

\subsection{Inhomogeneous double thinning approach}

The computation of the coverage probability in (3) is analytically tractable if the point process of the BSs is a PPP [11]. If it is not a PPP, on the other hand, the coverage probability cannot, in general, be formulated in a tractable analytical form [7]. To overcome this issue and enable one to analyze non-Poisson cellular networks, we have proposed the IDT approach in [7]. The essence of the approach consists of introducing an equivalent (approximated) network abstraction, for modeling non-Poisson cellular networks, that is based on inhomogeneous PPPs. The equivalent network model, in particular, is constituted by two independent inhomogeneous PPPs, $\Phi_{\mathrm{BS}}^{(F)}$ and $\Phi_{\mathrm{BS}}^{(K)}$, which are constructed with the purpose of approximating a motion-invariant point process from the point of view of the typical MT. Further details and discussions on the IDT approach and its interpretation from the typical MT standpoint can be found in [7].

Let the inhomogeneous PPPs $\Phi_{\mathrm{BS}}^{(F)}$ and $\Phi_{\mathrm{BS}}^{(K)}$ have intensity measures $\Lambda_{\Phi_{\mathrm{BS}}^{(F)}}(\cdot)$ and $\Lambda_{\Phi_{\mathrm{BS}}^{(K)}}(\cdot)$, respectively. The BS serving the probe MT is assumed to belong to $\Phi_{\mathrm{BS}}^{(F)}$, and the interfering BSs are assumed to belong to $\Phi_{\mathrm{BS}}^{(K)}$. In particular, the location of the serving BS and the inhomogeneous PPP, $\Phi_{\mathrm{BS}}^{(\mathrm{I})}$, of interfering BSs can be formulated as follows:

$$
\begin{aligned}
& x_{0}^{(F)}=\underset{x \in \Phi_{\mathrm{BS}}^{(F)}}{\arg \max }\{1 / l(x)\} \\
& \Phi_{\mathrm{BS}}^{(\mathrm{I})}=\Phi_{\mathrm{BS}}^{(\mathrm{I})}\left(x_{0}^{(F)}\right)=\left\{x \in \Phi_{\mathrm{BS}}^{(K)}: l(x)>L_{0}^{(F)}=l\left(x_{0}^{(F)}\right)\right\}
\end{aligned}
$$

It is worth mentioning, in particular, that the inhomogeneous PPPs $\Phi_{\mathrm{BS}}^{(F)}$ and $\Phi_{\mathrm{BS}}^{(\mathrm{I})}$ are only conditionally, i.e., upon $x_{0}^{(F)}$, independent. As described in detail in [7], the intensity measures of $\Phi_{\mathrm{BS}}^{(F)}$ and $\Phi_{\mathrm{BS}}^{(K)}$ are determined from the $F$-function and $K$-function of the original motioninvariant point process. Therefore, they depend only on the spatial characteristics of the point process (spatial model) being used. In [7], we have shown, in particular, that a convenient choice, which provides one with a good trade-off between modeling accuracy and analytical tractability, for the intensity measures $\Lambda_{\Phi_{B S}^{(F)}}(\cdot)$ and $\Lambda_{\Phi_{\mathrm{BS}}^{(K)}(\cdot)}$ is the following:

$$
\begin{aligned}
& \Lambda_{\Phi_{\mathrm{BS}}^{(F)}}(\mathcal{B}(0, r))=2 \pi \int_{0}^{r} \lambda_{\mathrm{BS}}^{(F)}(\zeta) \zeta d \zeta \\
& \Lambda_{\Phi_{\mathrm{BS}}^{(K)}}(\mathcal{B}(0, r))=2 \pi \int_{0}^{r} \lambda_{\mathrm{BS}}^{(K)}(\zeta) \zeta d \zeta
\end{aligned}
$$

where $\mathcal{B}(0, r)$ denotes the ball centered at the origin and of radius $r$ and $\lambda_{\mathrm{BS}}^{(F)}(\cdot)$ and $\lambda_{\mathrm{BS}}^{(K)}(\cdot)$ are the intensity functions of $\Phi_{\mathrm{BS}}^{(F)}$ and $\Phi_{\mathrm{BS}}^{(K)}$, respectively, which are distance-dependent and angle-independent.

In particular, $\lambda_{\mathrm{BS}}^{(F)}(\cdot)$ and $\lambda_{\mathrm{BS}}^{(K)}(\cdot)$ have different forms depending on whether the original motion-invariant point process is spatially repulsive or spatially attractive.

Spatially repulsive point process: If the point process $\Psi_{\mathrm{BS}}$ is spatially repulsive, then:

$$
\begin{aligned}
& \lambda_{\mathrm{BS}}^{(F)}(r)=\lambda_{\mathrm{BS}} \check{\check{c}}_{\mathrm{F}} \min \left\{\left(\check{\mathrm{a}}_{\mathrm{F}} / \check{\mathrm{c}}_{\mathrm{F}}\right) r+\check{\mathrm{b}}_{\mathrm{F}} / \check{\mathrm{c}}_{\mathrm{F}}, 1\right\} \\
& \lambda_{\mathrm{BS}}^{(K)}(r)=\lambda_{\mathrm{BS}} \min \left\{\check{\mathrm{a}}_{\mathrm{K}} r+\check{\mathrm{b}}_{\mathrm{K}}, \check{\mathrm{c}}_{\mathrm{K}}\right\}
\end{aligned}
$$

where $\left(\check{a}_{F}, \check{b}_{F}, \check{c}_{F}\right)$ and $\left(\check{a}_{K}, \check{b}_{K}, \check{c}_{K}\right)$ are two triplets of non-negative real numbers such that $\check{\mathrm{c}}_{\mathrm{F}} \geq \check{\mathrm{b}}_{\mathrm{F}} \geq 1$ and $\check{\mathrm{b}}_{\mathrm{K}} \leq \check{\mathrm{c}}_{\mathrm{K}} \leq 1$.

Spatially attractive point process: If the point process $\Psi_{\mathrm{BS}}$ is spatially attractive, then:

$$
\begin{aligned}
& \lambda_{\mathrm{BS}}^{(F)}(r)=\lambda_{\mathrm{BS}} \max \left\{-\hat{\mathrm{a}}_{\mathrm{F}} r+\hat{\mathrm{b}}_{\mathrm{F}}, \hat{\mathrm{c}}_{\mathrm{F}}\right\} \\
& \lambda_{\mathrm{BS}}^{(K)}(r)=\lambda_{\mathrm{BS}} \hat{\mathrm{b}}_{\mathrm{K}} \max \left\{-\left(\hat{\mathrm{a}}_{\mathrm{K}} / \hat{\mathrm{b}}_{\mathrm{K}}\right) r+1, \hat{\mathrm{c}}_{\mathrm{K}} / \hat{\mathrm{b}}_{\mathrm{K}}\right\}
\end{aligned}
$$

where $\left(\hat{a}_{F}, \hat{b}_{F}, \hat{c}_{F}\right)$ and $\left(\hat{a}_{K}, \hat{b}_{K}, \hat{c}_{K}\right)$ are two triplets of nonnegative real numbers such that $\hat{c}_{F} \leq \hat{b}_{F} \leq 1$ and $\hat{b}_{K} \geq$ $\hat{\mathrm{c}}_{\mathrm{K}} \geq 1$.

The intensity measures in (5) can be formulated in closed-form from (6) and (7), as described in [7]. Since for spatially repulsive and spatially attractive point processes the intensity measures in (5) have the same analytical expression as a function of the triplets of parameters, we use, in the sequel, the general notation $\left(\mathrm{a}_{\mathrm{F}}, \mathrm{b}_{\mathrm{F}}, \mathrm{c}_{\mathrm{F}}\right)$ and $\left(a_{K}, b_{K}, c_{K}\right)$ for both case studies.

\section{Meta distribution}

In this section, we introduce the meta distribution, overview the most common approaches for computing it, and show that it can be efficiently computed, within a given and bounded error, by using the trapezoidal integration rule and the Euler sum method. In what follows, we consider the equivalent network model based on the inhomogeneous PPPs $\Phi_{\mathrm{BS}}^{(F)}$ and $\Phi_{\mathrm{BS}}^{(K)}$. For ease of writing, we employ the notation $\Phi_{\mathrm{BS}}=\left\{\Phi_{\mathrm{BS}}^{(F)}, \Phi_{\mathrm{BS}}^{(K)}\right\}$.

\subsection{Definition}

According to [8], the meta distribution is defined as follows:

$$
\begin{aligned}
& \overline{\mathrm{F}}_{\mathrm{P}_{\text {cov }}}\left(\gamma_{\mathrm{D}}, \gamma_{\mathrm{A}}, z\right)=\operatorname{Pr}_{\Phi_{\mathrm{BS}}}\left\{\mathrm{P}_{\mathrm{cov}}\left(\gamma_{\mathrm{D}}, \gamma_{\mathrm{A}} \mid \Phi_{\mathrm{BS}}\right) \geq z\right\} \\
& \quad \text { with } z \in[0,1]
\end{aligned}
$$


where $\mathrm{P}_{\mathrm{cov}}\left(\gamma_{\mathrm{D}}, \gamma_{\mathrm{A}} \mid \Phi_{\mathrm{BS}}\right)$ is the coverage probability conditioned upon $\Phi_{\mathrm{BS}}$ (and by assuming that the typical MT is at the origin), which is defined as follows:

$P_{\text {cov }}\left(\gamma_{\mathrm{D}}, \gamma_{\mathrm{A}} \mid \Phi_{\mathrm{BS}}\right)=\operatorname{Pr}\left\{\operatorname{SIR} \geq \gamma_{\mathrm{D}}, \overline{\mathrm{SNR}} \geq \gamma_{\mathrm{A}} \mid \Phi_{\mathrm{BS}}\right\}$

It is worth mentioning that, in (8), we have emphasized that the probability is computed only with respect to $\Phi_{\mathrm{BS}}$.

The spatially averaged coverage probability in (2) can be retrieved from the meta distribution in (7) directly from its definition, as follows:

$\mathrm{P}_{\text {cov }}\left(\gamma_{\mathrm{D}}, \gamma_{\mathrm{A}}\right)=\mathbb{E}_{\Phi_{\mathrm{BS}}}\left\{\mathrm{P}_{\mathrm{cov}}\left(\gamma_{\mathrm{D}}, \gamma_{\mathrm{A}} \mid \Phi_{\mathrm{BS}}\right)\right\}=\int_{0}^{1} \overline{\mathrm{F}}_{\mathrm{P}_{\mathrm{cov}}}(z) d z$

In practical terms, the meta distribution provides one with the fraction of links whose SIR is greater than $\gamma_{D}$ and whose average SNR is greater than $\gamma_{\mathrm{A}}$ with probability at least equal to $z$ in each network realization. Therefore, it yields a more general statistical characterization of the performance of cellular networks beyond spatial averages.

\subsection{Computation: Gil-Pelaez method}

As discussed in [8], the direct computation of the meta distribution in (8) is not straightforward. A general approach to overcome this issue is to capitalize on the GilPelaez inversion theorem [15], which allows one to formulate the meta distribution as a function of the moments of the (conditional) coverage probability in (9).

In particular, the following holds [8]:

$$
\begin{aligned}
& \overline{\mathrm{F}}_{\mathrm{P}_{\mathrm{cov}}}\left(\gamma_{\mathrm{D}}, \gamma_{\mathrm{A}}, z\right)= \\
& \quad \frac{1}{2}+\frac{1}{\pi} \int_{0}^{+\infty} \frac{\operatorname{Im}\left\{\mathcal{M}_{j t}\left(\gamma_{\mathrm{D}}, \gamma_{\mathrm{A}}\right) \exp (-j t \ln (z))\right\}}{t} d t
\end{aligned}
$$

where $j=\sqrt{-1}$ is the imaginary unit, $\operatorname{Im}\{\cdot\}$ denotes the imaginary part operator, and $\mathcal{M}_{b}\left(\gamma_{\mathrm{D}}, \gamma_{\mathrm{A}}\right)$ is the $b$ th moment of the (conditional) coverage probability $\mathrm{P}_{\operatorname{cov}}\left(\gamma_{\mathrm{D}}, \gamma_{\mathrm{A}} \mid \Phi_{\mathrm{BS}}\right)$, which is defined as follows:

$$
\begin{aligned}
\mathcal{M}_{b}\left(\gamma_{\mathrm{D}}, \gamma_{\mathrm{A}}\right) & =\mathbb{E}_{\Phi_{\mathrm{BS}}}\left\{\left(\mathrm{P}_{\mathrm{cov}}\left(\gamma_{\mathrm{D}}, \gamma_{\mathrm{A}} \mid \Phi_{\mathrm{BS}}\right)\right)^{b}\right\} \\
& =\int_{0}^{1} b z^{b-1} \overline{\mathrm{F}}_{\mathrm{P}_{\mathrm{cov}}}(z) d z
\end{aligned}
$$

Therefore, the meta distribution can be obtained by first computing the moments of the (conditional) coverage probability $\mathrm{P}_{\mathrm{cov}}\left(\gamma_{\mathrm{D}}, \gamma_{\mathrm{A}} \mid \Phi_{\mathrm{BS}}\right)$ in (12) and by then computing the integral in (11). As remarked in [12-14], however, the computation of (11) is not always straightforward. Other methods need, in general, to be used instead, e.g., the Fourier-Jacobi expansion [12], and the Mnatsakanov's theorem $[13,14]$.

An alternative approach relies on approximating the meta distribution with another distribution. A nota- ble example is using the beta distribution for approximating it over the entire range of values $z \in[0,1]$. As remarked in [14], however, this approach cannot be applied if the actual distribution does not fulfill the class of the beta distribution. The following lemma shows, e.g., that this is the case if the coverage probability is defined in terms of SIR and $\overline{\mathrm{SNR}}$.

Lemma 1 Let $\left(\mathrm{a}_{\mathrm{F}}, \mathrm{b}_{\mathrm{F}}, \mathrm{c}_{\mathrm{F}}\right)$ be the generic triplet of parameters introduced in (6) and (7), and denote $\mathrm{d}_{\mathrm{F}}=$ $\left(\mathrm{c}_{\mathrm{F}}-\mathrm{b}_{\mathrm{F}}\right) / \mathrm{a}_{\mathrm{F}} \geq 0$. The meta distribution in (11) satisfies the following properties:

$$
\begin{aligned}
& \overline{\mathrm{F}}_{\mathrm{P}_{\text {cov }}}\left(\gamma_{\mathrm{D}}, \gamma_{\mathrm{A}}, z \rightarrow 0\right)= \\
& 1-\exp \left(-2 \pi \lambda_{\mathrm{BS}} \Psi\left(\left(\frac{\mathrm{P}_{\mathrm{tx}}}{\kappa \sigma_{\mathrm{N}}^{2} \gamma_{\mathrm{A}}}\right)^{1 / \gamma} ; \mathrm{a}_{\mathrm{F}}, \mathrm{b}_{\mathrm{F}}, \mathrm{c}_{\mathrm{F}}\right)\right) \\
& \overline{\mathrm{F}}_{\mathrm{P}_{\text {cov }}}\left(\gamma_{\mathrm{D}}, \gamma_{\mathrm{A}}, z \rightarrow 1\right)=0
\end{aligned}
$$

$$
\begin{aligned}
0 \leq & \overline{\mathrm{F}}_{\mathrm{P}_{\mathrm{cov}}}\left(\gamma_{\mathrm{D}}, \gamma_{\mathrm{A}}, z\right) \leq 1 \\
& \quad-\exp \left(-2 \pi \lambda_{\mathrm{BS}} \Psi\left(\left(\frac{\mathrm{P}_{\mathrm{tx}}}{\kappa \sigma_{\mathrm{N}}^{2} \gamma_{\mathrm{A}}}\right)^{1 / \gamma} ; \mathrm{a}_{\mathrm{F}}, \mathrm{b}_{\mathrm{F}}, \mathrm{c}_{\mathrm{F}}\right)\right)
\end{aligned}
$$

where $\Lambda_{\Phi_{\mathrm{BS}}^{(F)}}(\mathcal{B}(0, r))=2 \pi \lambda_{\mathrm{BS}} \Psi\left(r ; \mathrm{a}_{\mathrm{F}}, \mathrm{b}_{\mathrm{F}}, \mathrm{c}_{\mathrm{F}}\right)$, and:

$$
\begin{aligned}
\Psi\left(r ; \mathrm{a}_{\mathrm{F}}, \mathrm{b}_{\mathrm{F}}, \mathrm{c}_{\mathrm{F}}\right)= & \left(\frac{\mathrm{a}_{\mathrm{F}}}{3} r^{3}+\frac{\mathrm{b}_{\mathrm{F}}}{2} r^{2}\right) \mathbb{1}\left(r \leq \mathrm{d}_{\mathrm{F}}\right) \\
& +\left(\frac{\left(\mathrm{b}_{\mathrm{F}}-\mathrm{c}_{\mathrm{F}}\right)^{3}}{6 \mathrm{a}_{\mathrm{F}}^{2}}+\frac{\mathrm{c}_{\mathrm{F}}}{2} r^{2}\right) \mathbb{1}\left(r>\mathrm{d}_{\mathrm{F}}\right)
\end{aligned}
$$

\section{Proof See Appendix A.}

From Lemma 1, we evince that the meta distribution lies in the range $[0,1]$ only if $\gamma_{\mathrm{A}}=0$, i.e., the conventional definition of coverage probability based only on the SIR is used [11]. If $\gamma_{\mathrm{A}} \neq 0$, on the other hand, the meta distribution lies in the range $\left[0,1-\exp \left(-2 \pi \lambda_{\mathrm{BS}} \Psi\left(\left(\mathrm{P}_{\mathrm{tx}} /\left(\kappa \sigma_{\mathrm{N}}^{2} \gamma_{\mathrm{A}}\right)\right)^{1 / \gamma} ; \mathrm{a}_{\mathrm{F}}, \mathrm{b}_{\mathrm{F}}, \mathrm{c}_{\mathrm{F}}\right)\right)\right]$. This implies that the beta distribution is not necessarily a good approximation for the meta distribution, since the former distribution lies always in the range $[0,1]$.

\subsection{Computation: Euler sum method}

In this section, motivated by the considerations just made, we show that the meta distribution can be efficiently computed, with a known and bounded approximation error, by using the trapezoidal integration rule and the Euler sum method as originally proposed in [16] and recently used, e.g., in [17]. The following proposition states the result in rigorous terms. 
Proposition 1 Let $A, N$, and $Q$ be three positive integer numbers. Let us define the following functions:

$$
\begin{array}{ll}
\beta_{0}=2 & \\
\beta_{n}=1 \quad \text { for } & n=1,2, \ldots, N \\
s_{n}=\frac{A+2 \pi j n}{2} & \text { for } n=0,1, \ldots, N
\end{array}
$$

The meta distribution can be formulated as follows:

$$
\begin{aligned}
\overline{\mathrm{F}}_{\mathrm{P}_{\text {cov }}\left(\gamma_{\mathrm{D}}, \gamma_{\mathrm{A}}, z\right) \approx} & \frac{2^{-Q} \exp (A / 2)}{\ln ^{2}(z)} \sum_{q=0}^{Q}\left(\begin{array}{l}
Q \\
q
\end{array}\right) \\
& \sum_{n=0}^{N+q} \frac{(-1)^{n}}{\beta_{n}} \operatorname{Re}\left\{\frac{\mathcal{M}_{-s_{n} / \ln (z)}\left(\gamma_{\mathrm{D}}, \gamma_{\mathrm{A}}\right)}{s_{n}}\right\} \\
& +|\mathcal{E}(A, N, Q)|
\end{aligned}
$$

where $\operatorname{Re}\{\cdot\}$ is the real part operator, $\mathcal{M}_{b}\left(\gamma_{\mathrm{D}}, \gamma_{\mathrm{A}}\right)$ is the bth moment in (12), and $\mathcal{E}(A, N, Q)$ is the approximation error as follows:

$$
\begin{aligned}
|\mathcal{E}(A, N, Q)| \approx & \frac{1}{\exp (A)-1} \\
+ & \mid \frac{2^{-Q} \exp (A / 2)}{\ln ^{2}(z)} \sum_{q=0}^{Q}\left(\begin{array}{l}
Q \\
q
\end{array}\right)(-1)^{N+1+q} \\
& \operatorname{Re}\left\{\frac{\mathcal{M}_{-s_{N+1+q} / \ln (z)}\left(\gamma_{\mathrm{D}}, \gamma_{\mathrm{A}}\right)}{s_{N+1+q}}\right\} \mid
\end{aligned}
$$

Proof See Appendix B.

There are three main advantages in favor of using the Euler sum method instead of the Gil-Pelaez method: (1) Eq. 18 does not need the explicit computation of an integral, which makes the numerical estimation of the meta distribution easier; (2) the method can be applied to any family of non-negative meta distributions; and (3) the approximation error in (19) is known in closed-form and the accuracy of the numerical computation can be controlled by using the triplet of parameters $(A, N, Q)$. As discussed in [16], in particular, typical values of these parameters are $A=10 \ln (10)$, which guarantees a discretization error of the order of $10^{-10}$, and $N$ and $Q$ of the order of 10 or 20 .

\section{Computation of the moments}

From (18), it is apparent that the meta distribution can be easily obtained from the moments defined in (12). The following theorem provides one with these moments for the system model under analysis based on the IDT modeling approximation for non-Poisson cellular networks.
Theorem 1 Let $\left(\mathrm{a}_{\mathrm{F}}, \mathrm{b}_{\mathrm{F}}, \mathrm{c}_{\mathrm{F}}\right)$ and $\left(\mathrm{a}_{\mathrm{K}}, \mathrm{b}_{\mathrm{K}}, \mathrm{c}_{\mathrm{K}}\right)$ be the two triplets of parameters that quantify the spatial correlation properties of the point process that describes the locations of the cellular BSs. Let us define $\mathrm{d}_{\mathrm{F}}=\left(\mathrm{c}_{\mathrm{F}}-\mathrm{b}_{\mathrm{F}}\right) / \mathrm{a}_{\mathrm{F}} \geq 0$ and $\mathrm{d}_{\mathrm{K}}=\left(\mathrm{c}_{\mathrm{K}}-\mathrm{b}_{\mathrm{K}}\right) / \mathrm{a}_{\mathrm{K}} \geq 0$. The moments in (12) can be formulated as follows:

$$
\begin{aligned}
\mathcal{M}_{b}\left(\gamma_{\mathrm{D}}, \gamma_{\mathrm{A}}\right)= & \mathbb{E}_{\Phi_{\mathrm{BS}}}\left\{\left(\mathrm{P}_{\mathrm{Cov}}\left(\gamma_{\mathrm{D}}, \gamma_{\mathrm{A}} \mid \Phi_{\mathrm{BS}}\right)\right)^{b}\right\} \\
= & 2 \pi \lambda_{\mathrm{BS}} \int_{0}^{\left(\mathrm{P}_{\mathrm{tx}} /\left(\kappa \sigma_{\mathrm{N}}^{2} \gamma_{\mathrm{A}}\right)\right)^{1 / \gamma}} \\
& \exp \left(-2 \pi \lambda_{\mathrm{BS}} \Theta\left(r ; \gamma_{\mathrm{D}}\right)\right) \Upsilon(r) d r
\end{aligned}
$$

where the following functions are defined:

$$
\begin{aligned}
\Theta\left(r ; \gamma_{\mathrm{D}}\right) & =\frac{\mathrm{a}_{\mathrm{K}}}{3} r^{3}\left({ }_{2} F_{1}\left(b,-\frac{3}{\gamma}, 1-\frac{3}{\gamma}, \gamma_{\mathrm{D}}\right)-1\right) \mathbb{1}\left(r \leq \mathrm{d}_{\mathrm{K}}\right) \\
& +\frac{\mathrm{b}_{\mathrm{K}}}{2} r^{2}\left({ }_{2} F_{1}\left(b,-\frac{2}{\gamma}, 1-\frac{2}{\gamma}, \gamma_{\mathrm{D}}\right)-1\right) \mathbb{1}\left(r \leq \mathrm{d}_{\mathrm{K}}\right) \\
& -\frac{\mathrm{a}_{\mathrm{K}}}{3} \mathrm{~d}_{\mathrm{K}}^{3}\left({ }_{2} F_{1}\left(b,-\frac{3}{\gamma}, 1-\frac{3}{\gamma},-\frac{r^{\gamma}}{\mathrm{d}_{\mathrm{K}}^{\gamma}} \gamma_{\mathrm{D}}\right)-1\right) \mathbb{1}\left(r \leq \mathrm{d}_{\mathrm{K}}\right) \\
& +\frac{\mathrm{c}_{\mathrm{K}}-\mathrm{b}_{\mathrm{K}}}{2} \mathrm{~d}_{\mathrm{K}}^{2}\left({ }_{2} F_{1}\left(b,-\frac{2}{\gamma}, 1-\frac{2}{\gamma},-\frac{r^{\gamma}}{\mathrm{d}_{\mathrm{K}}^{\gamma}} \gamma_{\mathrm{D}}\right)-1\right) \mathbb{1}\left(r \leq \mathrm{d}_{\mathrm{K}}\right) \\
& +\frac{\mathrm{c}_{\mathrm{K}}}{2} r^{2}\left({ }_{2} F_{1}\left(b,-\frac{2}{\gamma}, 1-\frac{2}{\gamma},-\gamma_{\mathrm{D}}\right)-1\right) \mathbb{1}\left(r>\mathrm{d}_{\mathrm{K}}\right) \\
\Upsilon(r)= & \left(\mathrm{a}_{\mathrm{F}} r^{2}+\mathrm{b}_{\mathrm{F}} r\right) \exp \left(-2 \pi \lambda_{\mathrm{BS}}\left(\frac{\mathrm{a}_{\mathrm{F}}}{3} r^{3}+\frac{\mathrm{b}_{\mathrm{F}}}{2} r^{2}\right)\right) \mathbb{1}\left(r \leq \mathrm{d}_{\mathrm{F}}\right) \\
& +\mathrm{c}_{\mathrm{F}} r \exp \left(-2 \pi \lambda\left(\frac{\left(\mathrm{b}_{\mathrm{F}}-\mathrm{c}_{\mathrm{F}}\right)^{3}}{6 \mathrm{a}_{\mathrm{F}}^{2}}+\frac{\mathrm{c}_{\mathrm{F}}}{2} r^{2}\right)\right) \mathbb{1}\left(r>\mathrm{d}_{\mathrm{F}}\right)
\end{aligned}
$$

Proof See Appendix C.

The analytical framework in (20) can be applied to any non-Poisson spatial model that can be well approximated by using inhomogeneous PPPs with the aid of the IDT approach. The conventional Poisson cellular network model can be retrieved directly from Theorem 1 , as reported in the following corollary.

Corollary 1 In (homogeneous) Poisson cellular networks, the moments in (12) can be formulated as follows:

$$
\begin{aligned}
\mathcal{M}_{b}\left(\gamma_{\mathrm{D}}, \gamma_{\mathrm{A}}\right) \\
=\mathbb{E}_{\Phi_{\mathrm{BS}}\left\{\left(\mathrm{P}_{\mathrm{Cov}}\left(\gamma_{\mathrm{D}}, \gamma_{\mathrm{A}} \mid \Phi_{\mathrm{BS}}\right)\right)^{b}\right\}} \\
=\frac{1-\exp \left(-\pi \lambda_{\mathrm{BS}}\left(\frac{\mathrm{P}_{\mathrm{tx}}}{\kappa \sigma_{\mathrm{N}}^{2} \gamma_{\mathrm{A}}}\right)^{2 / \gamma}{ }_{2} F_{1}\left(b,-\frac{2}{\gamma}, 1-\frac{2}{\gamma},-\gamma_{\mathrm{D}}\right)\right)}{{ }_{2} F_{1}\left(b,-\frac{2}{\gamma}, 1-\frac{2}{\gamma},-\gamma_{\mathrm{D}}\right)}
\end{aligned}
$$


Proof It follows from (20) by setting $\mathrm{b}_{\mathrm{F}}=\mathrm{c}_{\mathrm{F}}=1$ and $\mathrm{b}_{\mathrm{K}}=\mathrm{c}_{\mathrm{K}}=1$, and by computing the integral.

By direct inspection of (23), in particular, we note that the moments reduce to those computed in [8] if $\gamma_{\mathrm{A}}=0$.

\section{Comparison between Poisson and non-Poisson cellular networks}

With the aid of the expression of the meta distribution in (18), and of its moments in (20) and (23) for non-Poisson and Poisson cellular networks, respectively, it is worth studying the impact of spatial correlations in cellular networks. By considering the spatially averaged definition of the coverage probability in (10), we have proved in [7], notably, that spatially repulsive and spatially attractive cellular networks provide better and worse coverage probability than Poisson cellular networks, respectively. Such a general result and comparison are, however, difficult to obtain for the meta distribution, since it can only be computed numerically, as shown in (18), and simple closed-form solutions for it are not available yet and are unknown. The following two propositions, however, provide one with some important information about this comparison.

Proposition 2 Let $z$ be sufficiently small, i.e., $z \rightarrow 0$. Let $\overline{\mathrm{F}}_{\mathrm{P}_{\text {cov }}}^{(\mathrm{PPP})}(\cdot), \overline{\mathrm{F}}_{\mathrm{P}_{\text {cov }}}^{\text {(Rep) }}(\cdot)$, and $\overline{\mathrm{F}}_{\mathrm{P}_{\text {cov }}}^{(\mathrm{Attr})}(\cdot)$ be the meta distributions of Poisson, spatially repulsive, and spatially attractive cellular networks, respectively. Then, the following holds:

$$
\begin{aligned}
& \overline{\mathrm{F}}_{\mathrm{P}_{\text {cov }}}^{(\mathrm{Rep})}\left(\gamma_{\mathrm{D}}, \gamma_{\mathrm{A}}, z \rightarrow 0\right) \geq \overline{\mathrm{F}}_{\mathrm{P}_{\text {cov }}}^{(\mathrm{PPP})}\left(\gamma_{\mathrm{D}}, \gamma_{\mathrm{A}}, z \rightarrow 0\right) \\
& \overline{\mathrm{F}}_{\mathrm{P}_{\text {cov }}}^{(\mathrm{Attr})}\left(\gamma_{\mathrm{D}}, \gamma_{\mathrm{A}}, z \rightarrow 0\right) \leq \overline{\mathrm{F}}_{\mathrm{P}_{\text {cov }}}^{(\mathrm{PPP})}\left(\gamma_{\mathrm{D}}, \gamma_{\mathrm{A}}, z \rightarrow 0\right)
\end{aligned}
$$

Proof Let $\Lambda_{\Phi_{\mathrm{BS}}^{(F)}}^{(\mathrm{PPP})}(\cdot), \Lambda_{\Phi_{\mathrm{BS}}^{(F)}}^{(\mathrm{Rep})}(\cdot)$, and $\Lambda_{\Phi_{\mathrm{BS}}^{(F)}}^{(\mathrm{Attr})}(\cdot)$ be the intensity measures in (5) for Poisson, spatially repulsive, and spatially attractive cellular networks, respectively. In [7, Lemma 5] and [7, Lemma 6], it is proved that $\Lambda_{\Phi_{\mathrm{BS}}^{(F)}}^{(\mathrm{Rep})}(\mathcal{B}(0, r)) \geq \Lambda_{\Phi_{\mathrm{BS}}^{(P)}}^{(\mathrm{PPP})}(\mathcal{B}(0, r))$ and $\Lambda_{\Phi_{\mathrm{BS}}^{(F)}}^{(\mathrm{PPP})}(\mathcal{B}(0, r)) \geq$ $\Lambda_{\Phi_{\mathrm{BS}}^{(F)}}^{(\mathrm{Att})}(\mathcal{B}(0, r))$, respectively. Then, the proof follows immediately from Lemma 1.

Proposition 3 Let $\quad \mathcal{M}_{b}^{(\mathrm{PPP})}(\cdot), \quad \mathcal{M}_{b}^{(\mathrm{Rep})}(\cdot), \quad$ and $\mathcal{M}_{b}^{\text {(Attr) }}(\cdot)$ be the bth moment in (12) for Poisson, spatially repulsive, and spatially attractive cellular networks, respectively. Then, for every $b \in \mathbb{R}$, the following holds:

$$
\begin{aligned}
& \mathcal{M}_{b}^{(\mathrm{Rep})}\left(\gamma_{\mathrm{D}}, \gamma_{\mathrm{A}}\right) \geq \mathcal{M}_{b}^{(\mathrm{PPP})}\left(\gamma_{\mathrm{D}}, \gamma_{\mathrm{A}}\right) \\
& \mathcal{M}_{b}^{(\mathrm{PPP})}\left(\gamma_{\mathrm{D}}, \gamma_{\mathrm{A}}\right) \geq \mathcal{M}_{b}^{(\mathrm{Attr})}\left(\gamma_{\mathrm{D}}, \gamma_{\mathrm{A}}\right)
\end{aligned}
$$

Proof See Appendix D.
Proposition 2, notably, allows one to conclude that, for low values of $z$ that tends to zero, spatially repulsive cellular networks exhibit first-order stochastic dominance over Poisson cellular networks, and that Poisson cellular networks exhibit first-order stochastic dominance over spatially attractive cellular networks. By using similar arguments, Proposition 3 allows one to establish similar moments-based stochastic ordering between Poisson and non-Poisson cellular networks.

\section{Numerical results and validation}

In this section, we show some simulation results in order to substantiate the main findings of the paper. The simulation setup is reported in Table 1, and the specific parameters of the point processes are detailed in [7]. The spatial model denoted by "general case" is chosen in order to better compare homogeneous, spatially attractive, and spatially repulsive point processes.

In Fig. 1, we report the moments of the conditional coverage probability. We observe a good agreement between the proposed analytical framework and Monte Carlo simulations. We note, in addition, that the findings about the first-order stochastic dominance of the moments is confirmed by our numerical illustrations.

In Figs. 2 and 3, we compare the meta distribution by considering two definitions of coverage probability. The conventional SIR-based definition and the SIR + SNRbased definition that is employed in this paper [11]. We observe that, in both cases, we obtain a good agreement compared with Monte Carlo simulations. The figures confirm, in addition, that the beta distribution may not be used for approximating the SIR $+\overline{\mathrm{SNR}}$-based definition of coverage probability. Our numerical results, on the other hand, confirm (even though they are not shown for ease of illustration) that the beta distribution yields a good approximation for the SIR-based definition of coverage probability.

In Fig. 4, finally, we compare the meta distribution of homogeneous, spatially attractive, and spatially repulsive point processes. We observe a good agreement with Monte Carlo simulations. In addition, the figures confirms the correctness of the asymptotic value of the meta distribution for small values of $z$.

\section{Conclusion and discussion}

In this paper, we have proved that the inhomogeneous double thinning approach for modeling and analyzing spatially correlated (non-Poisson) cellular networks can be successfully employed for studying the distribution of the conditional coverage probability given the point process of cellular base stations, which is referred to as the meta distribution. We have proved, in addition, that the meta distribution can be efficiently computed by using the trapezoidal integration rule and the Euler 
Table 1 Simulation setup

\begin{tabular}{|c|c|}
\hline Parameter & Value \\
\hline $\begin{array}{l}\text { Ginibre point process } \\
\text { (rural) }\end{array}$ & $\lambda_{\mathrm{BS}}=0.03056 \mathrm{BSs} / \mathrm{km}^{2}$ \\
\hline $\begin{array}{l}\text { Log-Gaussian Cox point } \\
\text { process (urban) }\end{array}$ & $\lambda_{\mathrm{BS}}=4.00923 \mathrm{BSs} / \mathrm{km}^{2}$ \\
\hline General case & $\lambda_{\mathrm{BS}}=0.2346 \mathrm{BSs} / \mathrm{km}^{2}$ \\
\hline $\begin{array}{l}\text { Ginibre point process } \\
\text { (rural) }\end{array}$ & $\beta=0.225$, Area region $=124.578 \pi \mathrm{km}^{2}$ \\
\hline $\begin{array}{l}\text { Log-Gaussian Cox point } \\
\text { process (urban) }\end{array}$ & $\begin{array}{l}\beta=0.03, \sigma^{2}=3.904, \mu=-0.5634 \\
\text { Area region }=28 \times 28 \mathrm{~km}^{2}\end{array}$ \\
\hline \multirow[t]{2}{*}{$\begin{array}{l}\text { Path-loss constant and } \\
\text { exponent }\end{array}$} & $\kappa=\left(4 \pi f_{c} / 3 \times 10^{8}\right)^{2}, \gamma=4$ \\
\hline & $a_{F}=4.55473414133037 \cdot 10^{-5}$ \\
\hline \multirow{3}{*}{$\begin{array}{l}\text { Ginibre point process } \\
\text { (rural) }\end{array}$} & $b_{F}=1.01046879386340$ \\
\hline & $C_{F}=1.11306423054186$ \\
\hline & $a_{K}=0.000400570907629641$ \\
\hline \multirow{3}{*}{$\begin{array}{l}\text { Ginibre point process } \\
\text { (rural) }\end{array}$} & $b_{K}=0.0118898483733152$ \\
\hline & $c_{K}=0.999999810503409$ \\
\hline & $a_{\mathrm{F}}=3.00375582041718 \cdot 10^{-3}$ \\
\hline \multirow{3}{*}{$\begin{array}{l}\text { Log-Gaussian Cox point } \\
\text { process (urban) }\end{array}$} & $b_{F}=0.999992970565002$ \\
\hline & $c_{F}=0.660720583433523$ \\
\hline & $a_{\mathrm{F}}=0.254520540961994 \cdot 10^{-3}$ \\
\hline \multirow{2}{*}{$\begin{array}{l}\text { Log-Gaussian Cox point } \\
\text { process (urban) }\end{array}$} & $b_{K}=1.17267857020013$ \\
\hline & $c_{K}=1.00000033357904$ \\
\hline \multirow{2}{*}{ General case (repulsive) } & $a_{\mathrm{F}}=0.2 \cdot 10^{-3}, b_{\mathrm{F}}=1.1, c_{\mathrm{F}}=1.5$ \\
\hline & $a_{K}=0.2 \cdot 10^{-3}, b_{K}=0.8, c_{K}=0.99$ \\
\hline \multirow{2}{*}{ General case (attractive) } & $a_{\mathrm{F}}=0.2 \cdot 10^{-3}, b_{\mathrm{F}}=0.99, c_{\mathrm{F}}=0.8$ \\
\hline & $a_{K}=0.2 \cdot 10^{-3}, b_{K}=1.5, c_{K}=1.1$ \\
\hline SIR and SNR thresholds & $\gamma_{D}=1, \gamma_{A}=1$ \\
\hline $\begin{array}{l}\text { Ginibre point process } \\
\text { (rural) }\end{array}$ & $\mathrm{P}_{\mathrm{tx}}=55 \mathrm{dBm}$ \\
\hline $\begin{array}{l}\text { Log-Gaussian Cox point } \\
\text { process (urban) }\end{array}$ & $P_{t x}=20 \mathrm{dBm}$ \\
\hline General case & $P_{t x}=15 \mathrm{dBm}$ \\
\hline $\begin{array}{l}\text { Ginibre point process } \\
\text { (rural) }\end{array}$ & $\mathrm{BW}=200 \mathrm{kHz}$ \\
\hline $\begin{array}{l}\text { Log-Gaussian Cox point } \\
\text { process (urban) }\end{array}$ & $\mathrm{BW}=200 \mathrm{kHz}$ \\
\hline General case & $\mathrm{BW}=2000 \mathrm{kHz}$ \\
\hline Noise power & $\sigma_{\mathrm{N}}^{2}=-174+10 \log _{10}(\mathrm{BW})+10 \mathrm{dBm}$ \\
\hline
\end{tabular}

sum method, provided that the negative moments of the conditional coverage probability can be computed. By using the inhomogeneous double thinning approach, it is proved that these latter moments can be formulated in terms of a single integral expression. Finally, some results on the first-order stochastic dominance of non-Poisson cellular networks over Poisson cellular networks are proved.

\section{Appendix A: Proof of Lemma 1}

By conditioning upon the point process $\Phi_{B S}$, the SIR is a random variable and the average SNR is a constant. Therefore, the (conditional) coverage probability can be formulated as follows:

$$
\begin{aligned}
P_{\text {Cov }} & \left(\gamma_{\mathrm{D}}, \gamma_{\mathrm{A}} \mid \Phi_{\mathrm{BS}}\right) \\
& =\operatorname{Pr}\left\{\operatorname{SIR} \geq \gamma_{\mathrm{D}}, \overline{\mathrm{SNR}} \geq \gamma_{\mathrm{A}} \mid \Phi_{\mathrm{BS}}\right\} \\
& =\left\{\begin{array}{l}
\operatorname{Pr}\left\{\mathrm{SIR} \geq \gamma_{\mathrm{D}} \mid \Phi_{\mathrm{BS}}\right\} \quad \text { if } \quad r_{0} \leq\left(\frac{\mathrm{P}_{\mathrm{tx}}}{\kappa \sigma_{\mathrm{N}}^{2} \gamma_{\mathrm{A}}}\right)^{1 / \gamma} \\
0 \text { otherwise }
\end{array}\right.
\end{aligned}
$$

where $r_{0}=\left\|x_{0}\right\|$ is the distance of the serving BS from the origin.

By definition of meta distribution, i.e., $\overline{\mathrm{F}}_{\mathrm{P}_{\text {cov }}}\left(\gamma_{\mathrm{D}}, \gamma_{\mathrm{A}}, z\right)=$ $\operatorname{Pr}_{\Phi_{\mathrm{BS}}}\left\{\mathrm{P}_{\mathrm{Cov}}\left(\gamma_{\mathrm{D}}, \gamma_{\mathrm{A}} \mid \Phi_{\mathrm{BS}}\right) \geq z\right\}$, and by letting $z \rightarrow 0$, we obtain:

$$
\begin{aligned}
& \overline{\mathrm{F}}_{\mathrm{P}_{\text {cov }}}\left(\gamma_{\mathrm{D}}, \gamma_{\mathrm{A}}, z \rightarrow 0\right) \\
& =\operatorname{Pr}_{\Phi_{\mathrm{BS}}}\left\{\mathrm{P}_{\text {Cov }}\left(\gamma_{\mathrm{D}}, \gamma_{\mathrm{A}} \mid \Phi_{\mathrm{BS}}\right) \geq z \rightarrow 0\right\} \\
& = \begin{cases}1 & \text { if } \quad r_{0} \leq\left(\frac{\mathrm{P}_{\mathrm{tx}}}{\kappa \sigma_{\mathrm{N}}^{2} \gamma_{\mathrm{A}}}\right)^{1 / \gamma} \\
0 & \text { otherwise }\end{cases} \\
& =\operatorname{Pr}\left\{r_{0} \leq\left(\frac{\mathrm{P}_{\mathrm{tx}}}{\kappa \sigma_{\mathrm{N}}^{2} \gamma_{\mathrm{A}}}\right)^{1 / \gamma}\right\} \\
& =1-\exp \left(-\Lambda_{\Phi_{\mathrm{BS}}^{(F)}}\left(\mathcal{B}\left(0,\left(\frac{\mathrm{P}_{\mathrm{tx}}}{\kappa \sigma_{\mathrm{N}}^{2} \gamma_{\mathrm{A}}}\right)^{1 / \gamma}\right)\right)\right)
\end{aligned}
$$

where the last identity is obtained from the definition of F-function of inhomogeneous PPPs.

The proof follows by computing (5) from (6) and (7).

\section{Appendix B: Proof of Proposition 1}

By definition of meta distribution, the following holds true:

$$
\begin{aligned}
\overline{\mathrm{F}}_{\mathrm{P}_{\text {cov }}}\left(\gamma_{\mathrm{D}}, \gamma_{\mathrm{A}}, z\right) & =\operatorname{Pr}_{\Phi_{\mathrm{BS}}}\left\{\mathrm{P}_{\mathrm{cov}}\left(\gamma_{\mathrm{D}}, \gamma_{\mathrm{A}} \mid \Phi_{\mathrm{BS}}\right) \geq z\right\} \\
& =\operatorname{Pr}_{\Phi_{\mathrm{BS}}}\left\{\ln \left(\mathrm{P}_{\mathrm{cov}}\left(\gamma_{\mathrm{D}}, \gamma_{\mathrm{A}} \mid \Phi_{\mathrm{BS}}\right)\right) \geq \ln (z)\right\} \\
& =\operatorname{Pr}_{\Phi_{\mathrm{BS}}}\left\{-\ln \left(\mathrm{P}_{\mathrm{Cov}}\left(\gamma_{\mathrm{D}}, \gamma_{\mathrm{A}} \mid \Phi_{\mathrm{BS}}\right) \leq-\ln (z)\right\}\right. \\
& =\operatorname{Pr}_{\Phi_{\mathrm{BS}}}\left\{Y_{\Phi_{\mathrm{BS}}} \leq-\ln (z)\right\}
\end{aligned}
$$

where $Y_{\Phi_{\mathrm{BS}}}=-\ln \left(\mathrm{P}_{\mathrm{Cov}}\left(\gamma_{\mathrm{D}}, \gamma_{\mathrm{A}} \mid \Phi_{\mathrm{BS}}\right)\right)$.

Therefore, the meta distribution can be computed by using the Euler sum method in [16] from the Laplace transform of the random variable $Y_{\Phi_{\mathrm{BS}}}$. In particular, the latter Laplace transform can be formulated as follows: 


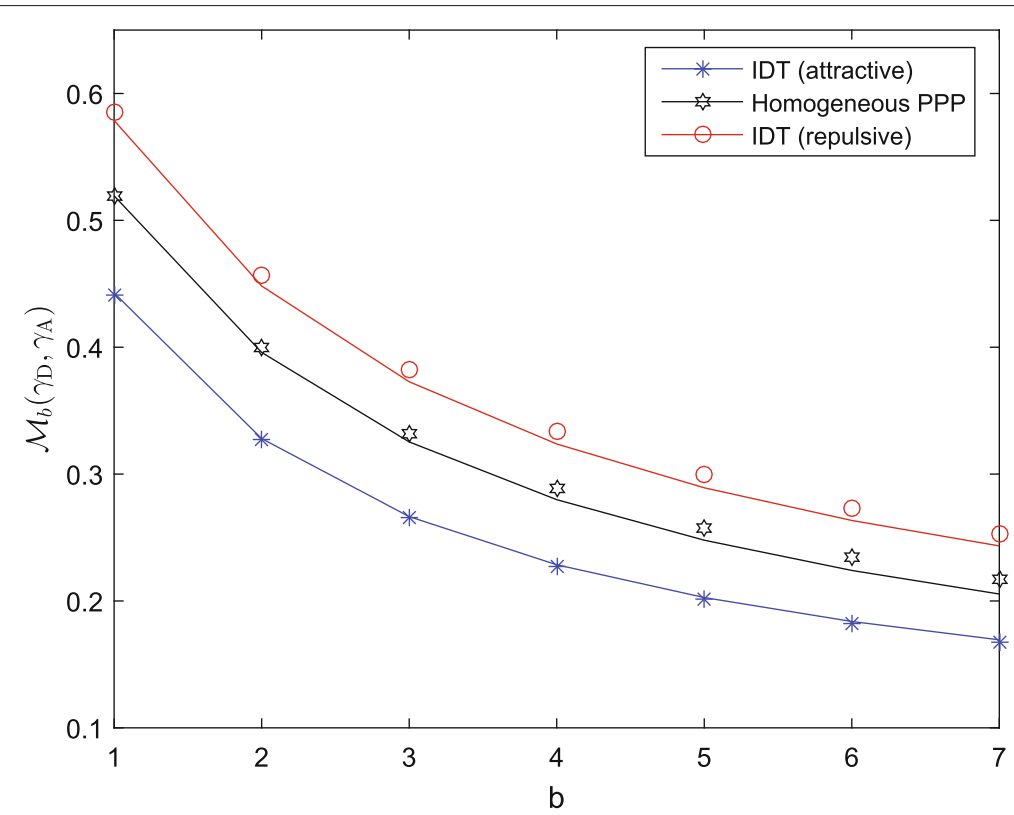

Fig. 1 Moments of the conditional coverage probability. Setup: General case. Solid lines: Monte Carlo simulations. Markers: IDT framework

$$
\begin{aligned}
\mathcal{L}_{Y_{\Phi_{\mathrm{BS}}}}(b) & =\mathbb{E}_{Y_{\Phi_{\mathrm{BS}}}}\left\{\exp \left(b Y_{\Phi_{\mathrm{BS}}}\right)\right\} \\
& =\mathbb{E}_{\Phi_{\mathrm{BS}}}\left\{\exp \left(-b \ln \left(\mathrm{P}_{\mathrm{Cov}}\left(\gamma_{\mathrm{D}}, \gamma_{\mathrm{A}} \mid \Phi_{\mathrm{BS}}\right)\right)\right)\right\} \\
& =\mathbb{E}_{\Phi_{\mathrm{BS}}}\left\{\left(\mathrm{P}_{\mathrm{Cov}}\left(\gamma_{\mathrm{D}}, \gamma_{\mathrm{A}} \mid \Phi_{\mathrm{BS}}\right)\right)^{-b}\right\} \\
& =\mathcal{M}_{-b}\left(\gamma_{\mathrm{D}}, \gamma_{\mathrm{A}}\right)
\end{aligned}
$$

which implies that the meta distribution is determined by the negative moments of the (conditional) coverage probability. Then, the proof follows.

\section{Appendix C: Proof of Theorem 1}

By definition, the $b$ th moment of the (conditional) coverage probability is the following:

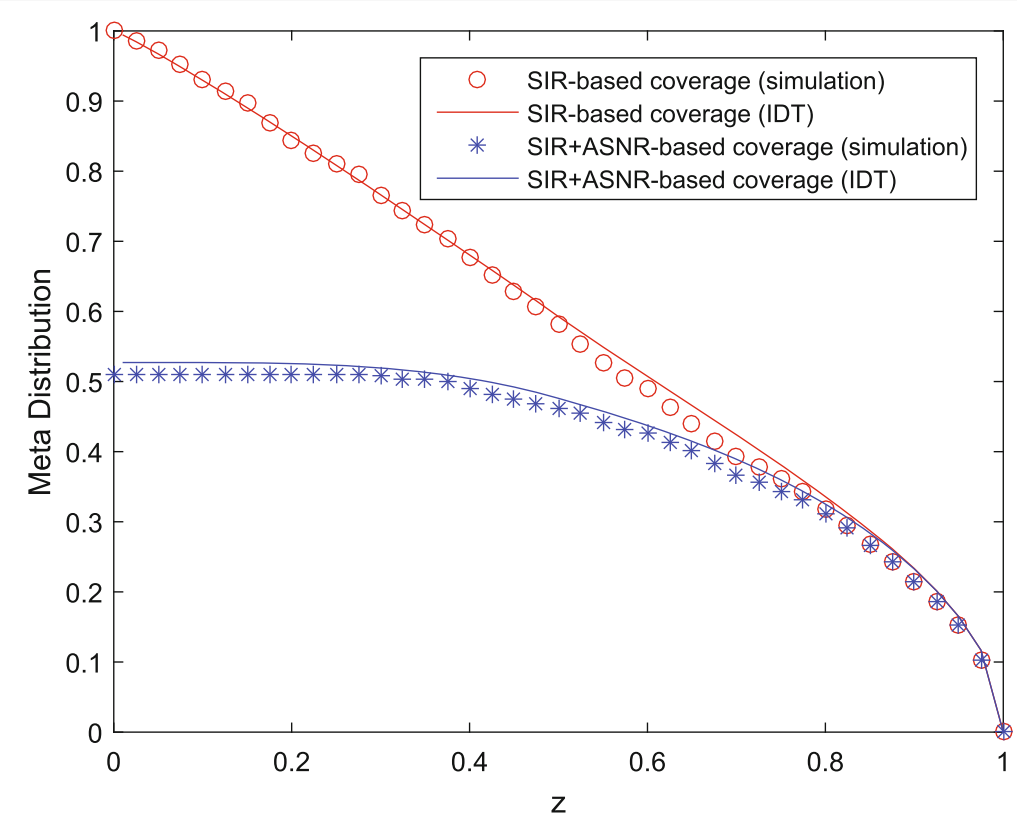

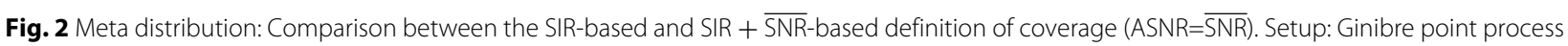
case. Solid lines: IDT framework. Markers: Monte Carlo simulations 


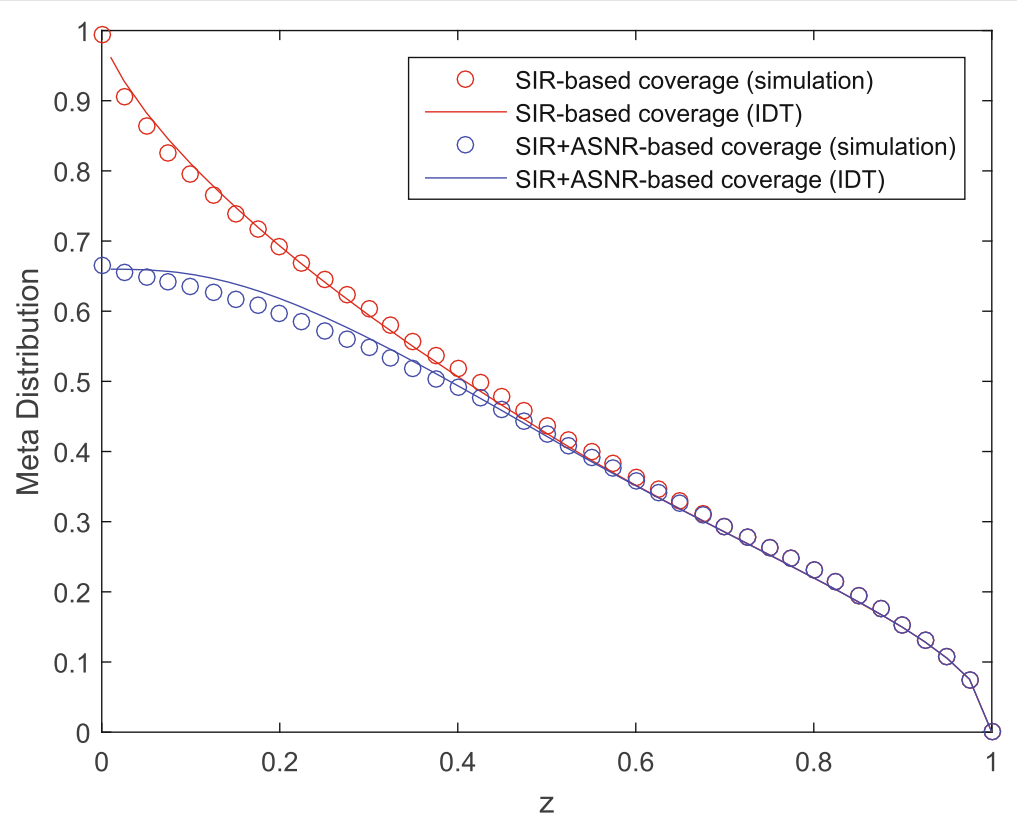

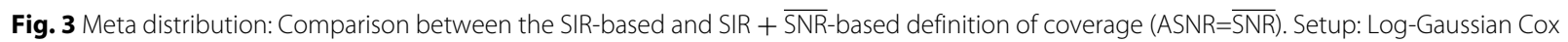
point process case. Solid lines: IDT framework. Markers: Monte Carlo simulations

$$
\mathcal{M}_{b}\left(\gamma_{\mathrm{D}}, \gamma_{\mathrm{A}}\right)=\mathbb{E}_{\Phi_{\mathrm{BS}}}\left\{\left(\mathrm{P}_{\mathrm{cov}}\left(\gamma_{\mathrm{D}}, \gamma_{\mathrm{A}} \mid \Phi_{\mathrm{BS}}\right)\right)^{b}\right\}
$$

By definition of (conditional) coverage probability, we obtain the following:

$$
\begin{aligned}
\operatorname{P}_{\operatorname{cov}}\left(\gamma_{\mathrm{D}}, \gamma_{\mathrm{A}} \mid \Phi_{\mathrm{BS}}\right) & =\operatorname{Pr}\left\{\operatorname{SIR} \geq \gamma_{\mathrm{D}}, \overline{\mathrm{SNR}} \geq \gamma_{\mathrm{A}} \mid \Phi_{\mathrm{BS}}\right\} \\
& =\operatorname{Pr}\left\{\operatorname{SIR} \geq \gamma_{\mathrm{D}} \mid \Phi_{\mathrm{BS}}\right\} \mathbb{1}\left(r_{0} \leq\left(\frac{\mathrm{P}_{\mathrm{tx}}}{\kappa \sigma_{\mathrm{N}}^{2} \gamma_{\mathrm{A}}}\right)^{1 / \gamma}\right) \\
& =\mathbb{1}\left(r_{0} \leq\left(\frac{\mathrm{P}_{\mathrm{tx}}}{\kappa \sigma_{\mathrm{N}}^{2} \gamma_{\mathrm{A}}}\right)^{1 / \gamma}\right) \prod_{x \in \Phi_{\mathrm{BS}}^{(K)}} v\left(\frac{r_{0}}{r}\right)
\end{aligned}
$$

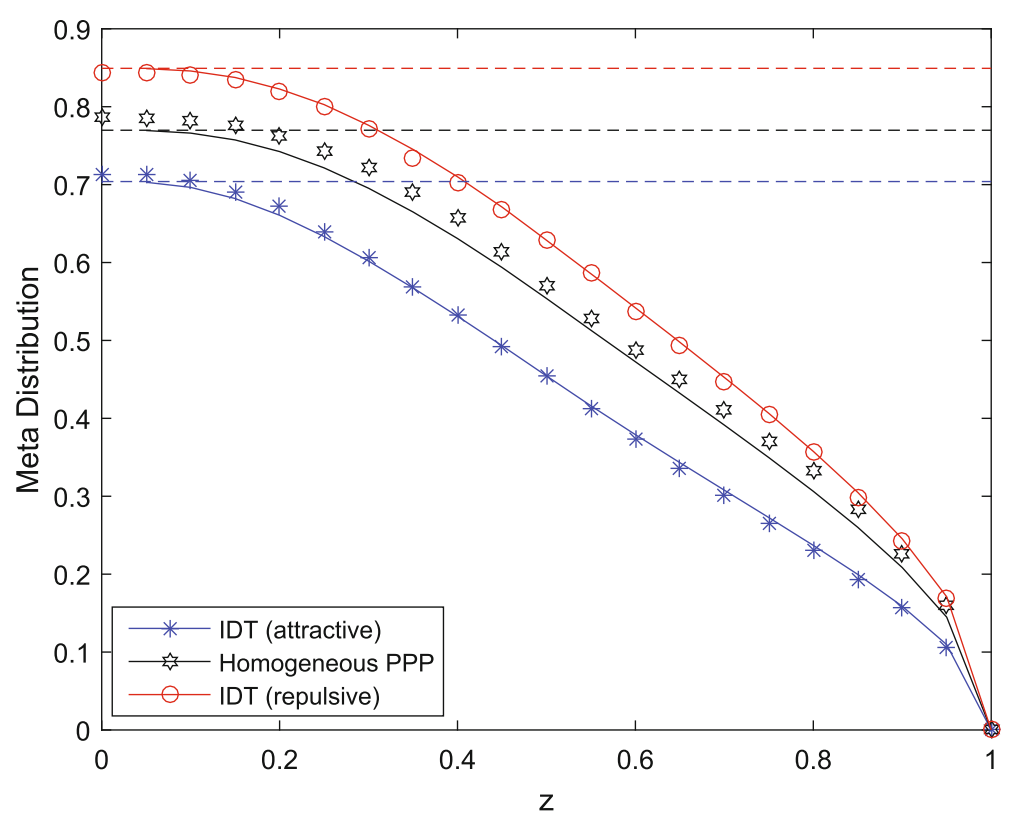

Fig. 4 Meta distribution. Setup: General case. Solid lines: IDT framework. Markers: Monte Carlo simulations. Dashed lines: asymptotic limit for $z \rightarrow 0$ 
where $r_{0}=\left\|x_{0}\right\|, r=\|x\|$, and $v(\xi)=\left(1+\gamma_{\mathrm{D}} \xi^{\gamma}\right)^{-b}$.

Therefore, the moments can be written as follows:

$$
\begin{aligned}
\mathcal{M}_{b}\left(\gamma_{\mathrm{D}}, \gamma_{\mathrm{A}}\right) & \\
= & \mathbb{E}_{\Phi_{\mathrm{BS}}^{(F)}}\left\{1\left(r_{0} \leq\left(\frac{\mathrm{P}_{\mathrm{tx}}}{\kappa \sigma_{\mathrm{N}}^{2} \gamma_{\mathrm{A}}}\right)^{1 / \gamma}\right) \mathbb{E}_{\Phi_{\mathrm{BS}}^{(K)}}\left\{\prod_{x \in \Phi_{\mathrm{BS}}^{(K)}} v\left(\frac{r_{0}}{r}\right)\right\}\right\} \\
= & \int_{0}^{\left(P_{\mathrm{tx}} /\left(\kappa \sigma_{\mathrm{N}}^{2} \gamma_{\mathrm{A}}\right)\right)^{1 / \gamma}} \mathbb{E}_{\Phi_{\mathrm{BS}}^{(K)}}\left\{\prod_{x \in \Phi_{\mathrm{BS}}^{(K)}} v\left(\frac{r_{0}}{r}\right)\right\} \\
& \times \Lambda_{\Phi_{\mathrm{BS}}^{(F)}}^{(1)}\left(\mathcal{B}\left(0, r_{0}\right)\right) \exp \left(-\Lambda_{\Phi_{\mathrm{BS}}^{(F)}}\left(\mathcal{B}\left(0, r_{0}\right)\right)\right) d r_{0}
\end{aligned}
$$

where the last equality follows by applying the void probability theorem of inhomogeneous PPPs, $\Lambda_{\Phi_{\mathrm{BS}}^{(F)}}^{(1)}(\mathcal{B}(0, r))$ is the first-order derivative of $\Lambda_{\Phi_{\mathrm{BS}}^{(F)}}(\mathcal{B}(0, r))$ computed with respect to $r$, and, by using the probability generating functional theorem, we have:

$$
\begin{aligned}
\mathbb{E}_{\Phi_{\mathrm{BS}}^{(K)}} & \left\{\prod_{x \in \Phi_{\mathrm{BS}}^{(K)}} v\left(\frac{r_{0}}{r}\right)\right\} \\
& =\exp \left(-\int_{r_{0}}^{+\infty}\left(1-v\left(\frac{r_{0}}{r}\right)\right) \Lambda_{\Phi_{\mathrm{BS}}^{(K)}}^{(1)}(\mathcal{B}(0, r)) d r\right)
\end{aligned}
$$

where $\Lambda_{\Phi_{\mathrm{BS}}^{(K)}}^{(1)}(\mathcal{B}(0, r))$ is the first-order derivative of $\Lambda_{\Phi_{\mathrm{BS}}^{(K)}}(\mathcal{B}(0, r))$ computed with respect to $r$.

The proof follows by computing the integrals and using the same steps are those reported in [7].

\section{Appendix D: Proof of Proposition 3}

For simplicity, we consider the case study of spatially repulsive cellular networks. A similar approach can be used to prove Proposition 3 if the cellular network is spatially repulsive.

From Appendix C, the moments of the (conditional) coverage probability can be formumated as follows:

$$
\mathcal{M}_{b}^{(\mathrm{Rep})}\left(\gamma_{\mathrm{D}}, \gamma_{\mathrm{A}}\right)=\int_{0}^{\left(\mathrm{P}_{\mathrm{tx}} /\left(\kappa \sigma_{\mathrm{N}}^{2} \gamma_{\mathrm{A}}\right)\right)^{1 / \gamma}} \mathcal{G}^{(\mathrm{Rep})}\left(r_{0}\right) \mathcal{H}^{(\mathrm{Rep})}\left(r_{0}\right) d r_{0}
$$

where the following shorthand notation is used:

$$
\mathcal{G}^{(\mathrm{Rep})}\left(r_{0}\right)=\exp \left(-\int_{r_{0}}^{+\infty}\left(1-v\left(\frac{r_{0}}{r}\right)\right) \Lambda_{\Phi_{\mathrm{BS}}^{(K)}}^{(1)}(\mathcal{B}(0, r)) d r\right)
$$

$$
\mathcal{H}^{(\mathrm{Rep})}\left(r_{0}\right)=\Lambda_{\Phi_{\mathrm{BS}}^{(F)}}^{(1)}\left(\mathcal{B}\left(0, r_{0}\right)\right) \exp \left(-\Lambda_{\Phi_{\mathrm{BS}}^{(F)}}\left(\mathcal{B}\left(0, r_{0}\right)\right)\right)
$$

Similar analytical expressions can be obtained for homogenous PPPs. In particular, the following holds true:

$$
\mathcal{M}_{b}^{(\mathrm{PPP})}\left(\gamma_{\mathrm{D}}, \gamma_{\mathrm{A}}\right)=\int_{0}^{\left(\mathrm{P}_{\mathrm{tx}} /\left(\kappa \sigma_{\mathrm{N}}^{2} \gamma_{\mathrm{A}}\right)\right)^{1 / \gamma}} \mathcal{G}^{(\mathrm{PPP})}\left(r_{0}\right) \mathcal{H}^{(\mathrm{PPP})}\left(r_{0}\right) d r_{0}
$$

where:

$$
\mathcal{G}^{(\mathrm{PPP})}\left(r_{0}\right)=\exp \left(-\pi \lambda_{\mathrm{BS}} r_{0}^{2}\left({ }_{2} F_{1}\left(b,-\frac{2}{\gamma} ; 1-\frac{2}{\gamma} ;-\gamma_{\mathrm{D}}\right)-1\right)\right)
$$

$$
\mathcal{H}^{(\mathrm{PPP})}\left(r_{0}\right)=2 \pi \lambda_{\mathrm{BS}} r_{0} \exp \left(-\pi \lambda_{\mathrm{BS}} r_{0}^{2}\right)
$$

Since $1-v\left(r_{0} / r\right) \geq 0$, and the inequality $\Lambda_{\Phi_{\mathrm{BS}}^{(K)}}^{(1)}\left(\mathcal{B}\left(0, r_{0}\right)\right) \leq \Lambda_{\mathrm{PPP}}^{(1)}\left(\mathcal{B}\left(0, r_{0}\right)\right)$ was proved in [7], then we have:

$$
\begin{aligned}
\mathcal{G}^{(\mathrm{Rep})} & \left(r_{0}\right) \\
& =\exp \left(-\int_{r_{0}}^{+\infty}\left(1-v\left(\frac{r_{0}}{r}\right)\right) \Lambda_{\Phi_{\mathrm{BS}}^{(K)}}^{(1)}(\mathcal{B}(0, r)) d r\right) \\
& \geq \exp \left(-\int_{r_{0}}^{+\infty}\left(1-v\left(\frac{r_{0}}{r}\right)\right) \Lambda_{\mathrm{PPP}}^{(1)}(\mathcal{B}(0, r)) d r\right) \\
& =\mathcal{G}^{(\mathrm{PPP})}\left(r_{0}\right)
\end{aligned}
$$

Therefore, we obtain the following:

$$
\begin{aligned}
\mathcal{M}_{b}^{(\mathrm{Rep})} & \left(\gamma_{\mathrm{D}}, \gamma_{\mathrm{A}}\right) \\
& =\int_{0}^{\left(\mathrm{P}_{\mathrm{tx}} /\left(\kappa \sigma_{\mathrm{N}}^{2} \gamma_{\mathrm{A}}\right)\right)^{1 / \gamma}} \mathcal{G}^{(\mathrm{Rep})}\left(r_{0}\right) \mathcal{H}^{(\mathrm{Rep})}\left(r_{0}\right) d r_{0} \\
& \geq \int_{0}^{\left(\mathrm{P}_{\mathrm{tx}} /\left(\kappa \sigma_{\mathrm{N}}^{2} \gamma_{\mathrm{A}}\right)\right)^{1 / \gamma}} \mathcal{G}^{(\mathrm{PPP})}\left(r_{0}\right) \mathcal{H}^{(\mathrm{Rep})}\left(r_{0}\right) d r_{0}
\end{aligned}
$$

Let us define $\underline{\mathcal{H}}^{(\mathrm{Rep})}\left(r_{0}\right)=1-\exp \left(-\Lambda_{\Phi_{\mathrm{BS}}^{(F)}}\left(\mathcal{B}\left(0, r_{0}\right)\right)\right)$. Then, we have the following:

$$
\begin{aligned}
\mathcal{J}= & \int_{0}^{\left(\mathrm{P}_{\mathrm{tx}} /\left(\kappa \sigma_{\mathrm{N}}^{2} \gamma_{\mathrm{A}}\right)\right)^{1 / \gamma}} \mathcal{G}^{(\mathrm{PPP})}\left(r_{0}\right) \mathcal{H}^{(\mathrm{Rep})}\left(r_{0}\right) d r_{0} \\
\stackrel{(a)}{=} & \left.\mathcal{G}^{(\mathrm{PPP})}\left(r_{0}\right) \underline{\mathcal{H}}^{(\mathrm{Rep})}\left(r_{0}\right)\right|_{0} ^{\left(\mathrm{P}_{\mathrm{tx}} /\left(\kappa \sigma_{\mathrm{N}}^{2} \gamma_{\mathrm{A}}\right)\right)^{1 / \gamma}} \\
& -\int_{0}^{\left(\mathrm{P}_{\mathrm{tx}} /\left(\kappa \sigma_{\mathrm{N}}^{2} \gamma_{\mathrm{A}}\right)\right)^{1 / \gamma}}\left(\frac{d \mathcal{G}^{(\mathrm{PPP})}\left(r_{0}\right)}{d r_{0}}\right) \underline{\mathcal{H}}^{(\mathrm{Rep})}\left(r_{0}\right) d r_{0} \\
& \stackrel{(b)}{=} \mathcal{G}^{(\mathrm{PPP})}\left(\left(\frac{\mathrm{P}_{\mathrm{tx}}}{\kappa \sigma_{\mathrm{N}}^{2} \gamma_{\mathrm{A}}}\right)^{1 / \gamma}\right) \underline{\mathcal{H}}^{(\mathrm{Rep})}\left(\left(\frac{\mathrm{P}_{\mathrm{tx}}}{\kappa \sigma_{\mathrm{N}}^{2} \gamma_{\mathrm{A}}}\right)^{1 / \gamma}\right) \\
& -\int_{0}^{\left(\mathrm{P}_{\mathrm{tx}} /\left(\kappa \sigma_{\mathrm{N}}^{2} \gamma_{\mathrm{A}}\right)\right)^{1 / \gamma}}\left(\frac{d \mathcal{G}^{(\mathrm{PPP})}\left(r_{0}\right)}{d r_{0}}\right) \underline{\mathcal{H}}^{(\mathrm{Rep})}\left(r_{0}\right) d r_{0}
\end{aligned}
$$


where (a) follows by applying the integration by parts rule, and (b) follows by taking into account that $\underline{\mathcal{H}}^{\text {(Rep) }}(0)=0$.

Since $\mathcal{G}^{(\mathrm{PPP})}\left(r_{0}\right) \geq 0, d \mathcal{G}^{(\mathrm{PPP})}\left(r_{0}\right) / d r_{0} \leq 0$, and it was proved in [7] that $\underline{\mathcal{H}}^{(\mathrm{Rep})}\left(r_{0}\right) \geq \underline{\mathcal{H}}^{(\mathrm{PPP})}\left(r_{0}\right)=1-$ $\exp \left(-\pi \lambda_{\mathrm{BS}} r_{0}^{2}\right)$, then we have the following:

$$
\begin{aligned}
& \mathcal{M}_{b}^{(\operatorname{Rep})}\left(\gamma_{\mathrm{D}}, \gamma_{\mathrm{A}}\right) \\
& \geq \int_{0}^{\left(\mathrm{P}_{\mathrm{tx}} /\left(\kappa \sigma_{\mathrm{N}}^{2} \gamma_{\mathrm{A}}\right)\right)^{1 / \gamma}} \mathcal{G}^{(\mathrm{PPP})}\left(r_{0}\right) \mathcal{H}^{(\mathrm{Rep})}\left(r_{0}\right) d r_{0} \\
& \geq \mathcal{G}^{(\mathrm{PPP})}\left(\left(\frac{\mathrm{P}_{\mathrm{tx}}}{\kappa \sigma_{\mathrm{N}}^{2} \gamma_{\mathrm{A}}}\right)^{1 / \gamma}\right) \underline{\mathcal{H}}^{(\mathrm{PPP})}\left(\left(\frac{\mathrm{P}_{\mathrm{tx}}}{\kappa \sigma_{\mathrm{N}}^{2} \gamma_{\mathrm{A}}}\right)^{1 / \gamma}\right) \\
& -\int_{0}^{\left(\mathrm{P}_{\mathrm{tx}} /\left(\kappa \sigma_{\mathrm{N}}^{2} \gamma_{\mathrm{A}}\right)\right)^{1 / \gamma}}\left(\frac{d \mathcal{G}^{(\mathrm{PPP})}\left(r_{0}\right)}{d r_{0}}\right) \underline{\mathcal{H}}^{(\mathrm{PPP})}\left(r_{0}\right) d r_{0} \\
& =\mathcal{M}_{b}^{(\mathrm{PPP})}\left(\gamma_{\mathrm{D}}, \gamma_{\mathrm{A}}\right)
\end{aligned}
$$

from which the proof follows.

\section{Funding}

This work was supported in part by the European Commission through the H2020-MSCA ETN-5Gwireless project under Grant Agreement 641985.

\section{Availability of data and materials}

Data sharing is not applicable to this article as no datasets were generated or analyzed during the current study. The paper is built upon mathematical analysis.

\section{Authors' contributions}

The authors declare that they have equally contributed to the paper. Both authors read and approved the final manuscript.

\section{Competing interests}

The authors declare that they have no competing interests.

\section{Publisher's Note}

Springer Nature remains neutral with regard to jurisdictional claims in published maps and institutional affiliations.

Received: 1 March 2019 Accepted: 25 April 2019

Published online: 17 June 2019

\section{References}

1. J. G. Andrews, F. Baccelli, R. K. Ganti, A tractable approach to coverage and rate in cellular networks. IEEE Trans. Commun. 59(11), 3122-3134 (2011)

2. M. Di Renzo, A. Guidotti, G. E. Corazza, Average rate of downlink heterogeneous cellular networks over generalized fading channels - A stochastic geometry approach. IEEE Trans. Commun. 61(7), 3050-3071 (2013)

3. M. Di Renzo, W. Lu, The equivalent-in-distribution (EiD)-based approach: On the analysis of cellular networks using stochastic geometry. IEEE Commun. Lett. 18(5), 761-764 (2014)

4. M. Di Renzo, W. Lu, Stochastic geometry modeling and performance evaluation of MIMO cellular networks using the equivalent-in-distribution (EiD)-based approach. IEEE Trans. Commun. 63(3), 977-996 (2015)

5. M. Di Renzo, Stochastic geometry modeling and analysis of multi-tier millimeter wave cellular networks. IEEE Trans. Wirel. Commun. 14(9), 5038-5057 (2015)

6. M. Di Renzo, W. Lu, P. Guan, The intensity matching approach: A tractable stochastic geometry approximation to system-level analysis of cellular networks. IEEE Trans. Wirel. Commun. 15(9), 5963-5983 (2016)
7. M. Di Renzo, S. Wang, X. Xi, Inhomogeneous double thinning - Modeling and analysis of cellular networks by using inhomogeneous Poisson point processes. IEEE Trans. Wirel. Commun. 17(8), 5162-5182 (2018)

8. M. Haenggi, The meta distribution of the SIR in Poisson bipolar and cellular networks. IEEE Trans. Wirel. Commun. 15(4), 2577-2589 (2016)

9. S. S. Kalamkar, M. Haenggi, Simple pproximations of the SIR meta distribution in general cellular networks. IEEE Trans. Commun. to appear. [Online]. Available: https://arxiv.org/pdf/1902.06457.pdf

10. M. Di Renzo, A. Zappone, T. T. Lam, M. Debbah, System-level modeling and optimization of the energy efficiency in cellular networks - A stochastic geometry framework. IEEE Trans. Wirel. Commun. 17(4), 2539-2556 (2018)

11. M. Di Renzo, T. T. Lam, A. Zappone, M. Debbah, A tractable closed-form expression of the coverage probability in poisson cellular networks. IEEE Wirel. Comm. Lett. 8(1), 249-252 (2019)

12. S. Guruacharya, E. Hossain, Approximation of meta distribution and its moments for Poisson cellular networks. ArXiv (2018). [Online]. Available: https://arxiv.org/abs/1804.06881

13. A. M. Hayajneh, S. A. R. Zaidi, D. C. McLernon, M. Z. Win, A. Imran, M. Ghogho, Optimal coverage and rate in downlink cellular networks: A SIR meta-distribution based approach. ArXiv (2018). [Online]. Available: https://arxiv.org/abs/1804.06881

14. M. Haenggi, Efficient calculation of meta distributions and the performance of user percentiles. IEEE Wirel. Commun. Lett. 7(6), 982-985 (2018)

15. M. Di Renzo, P. Guan, Stochastic geometry modeling of coverage and rate of cellular networks using the Gil-Pelaez inversion theorem. IEEE Commun. Lett. 18(9), 1575-1578 (2014)

16. J. Abate, W. Whitt, Numerical inversion of Laplace transforms of probability distribution. ORSA J. Comput. 7(1), 36-43 (1995)

17. X. Zhou, J. Guo, S. Durrani, M. Di Renzo, Power beacon-assisted millimeter wave ad hoc networks. IEEE Trans. Commun. 66(2), 830-844 (2018)

\section{Submit your manuscript to a SpringerOpen ${ }^{\circ}$ journal and benefit from:}

- Convenient online submission

- Rigorous peer review

- Open access: articles freely available online

- High visibility within the field

- Retaining the copyright to your article

Submit your next manuscript at $>$ springeropen.com 